\title{
Influence of Remained Medial Osteophyte on the Outcome after Ulnohumeral Arthroplasty
}

\section{Shingo Nobuta ${ }^{1^{*}}$, Katsumi Sato ${ }^{2}$ and Eiji Itoi ${ }^{3}$}

${ }^{1}$ Department of Orthopaedic Surgery, Tohoku Rosai Hospital, Sendai, Japan

${ }^{2}$ Tohoku Rosai Hospital, Sendai, Japan

${ }^{3}$ Department of Orthopaedic Surgery, Tohoku University School of Medicine, Sendai, Japan

*Corresponding author: Shingo Nobuta, Department of Orthopaedic Surgery, Tohoku Rosai Hospital 4-3-21, Dainohara, Aoba-ku, Sendai, Miyagi 981-8563, Japan, Tel: +81-22-275-1111; Fax: +81-22-275-7541; E-mail: nobutays@jc5.so-net.ne.jp

Received date: January 25, 2018; Accepted date: February 15, 2018; Published date: February 20, 2018

Copyright: (c) 2018 Nobuta S, et al. This is an open-access article distributed under the terms of the Creative Commons Attribution License, which permits unrestricted use, distribution, and reproduction in any medium, provided the original author and source are credited.

\begin{abstract}
Objective: The authors reported the long-term results of ulnohumeral arthroplasty (UHA) for symptomatic elbow osteoarthritis. UHA does not allow access to the medial ulnohumeral osteophyte and to the radiohumeral joint, and the influence of remained medial ulnohumeral osteophyte on the outcome is still unclear. The purpose of the present study was to determine the influence of remained medial osteophyte on the outcome in a much larger sample size.

Methods: Fifty-eight elbows in 51 patients with elbow osteoarthritis underwent UHA and were evaluated. Before surgery, all patients complained of motion pain and loss of flexion-extension arc of the elbow. Preoperative evaluation and the outcome at follow-up were assessed using Mayo Elbow Performance Score (MEPS), and medial ulnohumeral osteophyte was assessed in anteroposterior radiograph.
\end{abstract}

Results: Forty-one elbows had no pain and 17 decreased pain. The average flexion-extension arc improved from $93^{\circ}$ preoperatively to $107^{\circ}$ postoperatively. Total MEPS improved from 69 to 91 . The result was excellent for 40 elbows $(69 \%)$, good for $14(24 \%)$, fair for four $(7 \%)$ and no poor case. Patients' satisfaction showed that fifty $(86 \%)$ were satisfactory subjective result and eight $(14 \%)$ were unsatisfactory result. The preoperative flexion-extension arc was factors for predicting postoperative results. Remained medial ulnohumeral osteophyte was related to decreased arc of the flexion-extension but not patients' satisfaction and pain scale.

Conclusions: Remained medial ulnohumeral osteophyte was related to decreased arc of the flexion-extension but not patients' satisfaction and pain scale after UHA.

Keywords: Elbow; Osteoarthritis; Arthroplasty; Ulnohumeral joint; Functional outcome

\section{Introduction}

Primary osteoarthritis of the elbow is commonly caused in laborers and athletes, who heavily use the arm. Many elbow osteoarthritis can be treated conservatively, when conservative management fails, surgical treatment is indicated. In 1995, the authors started to employ ulnohumeral arthroplasty (UHA) [1] for elbow osteoarthritis, and reported the long-term results of twenty-two elbows [2], and described that 17 of 22 elbows had satisfactory results.

However, the surgical technique of UHA does not allow access to the medial ulnohumeral osteophyte and to the radiohumeral joint, and the influence of remained medial ulnohumeral osteophyte on the outcome is still unclear. Minami [3] and Oka et al. [4] reported that the main lesions of elbow osteoarthritis are present at the medial ulnohumeral joint which cause pain and limitation of motion. The purpose of the present study was to determine the influence of remained medial ulnohumeral osteophyte on the outcome in a larger sample size.

\section{Patients and Methods}

From 1999 to 2012, 58 elbows from 51 patients with osteoarthritis underwent UHA and followed up for 24-160 months (mean, 43 months). Forty-seven patients (seven bilateral) were men and four were women. The average age was 59 years (26 to 78$)$.

Thirty-nine patients with forty-six elbows (seven bilateral) were involved in a manual worker (ten farmers, eight carpenters, six mechanics, five pipe fitters, four electricians, four seamen, and two truck drivers), seven patients were office worker and five were unemployed. The dominant arm was involved in 45 elbows and the non-dominant in 13.

Before surgery, forty-five had terminal motion pain and thirteen had continuous elbow pain. Mean duration of symptoms was 49 months (range, 3-280 months). We measured extension, flexion, pronation, and supination of the elbow by a goniometer.

Fifty-six elbows had loss of extension more than $10^{\circ}$ and 42 had limited flexion less than $120^{\circ}$. The average flexion-extension arc before surgery was $19^{\circ}$ to $112^{\circ}$. We obtained written informed consent from all patients.

We used Mayo Elbow Performance Score (MEPS) [5] to evaluate elbow condition. The MEPS composed of 4 categories: pain (45 
Page 2 of 4

points), motion (20 points), stability (1 points) and function (25 points).

The MEPS objective evaluation of the elbow function is classified as excellent ( $>90$ points), good ( 75 to 89 points), fair (60 to 74 points) or poor ( $<60$ points).

Mean preoperative total MEPS score was 69 points (range, 55-75). The patients satisfaction was graded as much better or better (satisfactory), unchanged or worse (unsatisfactory) [6]. The preoperative radiographs showed olecranon osteophyte (bone spur protrusion by $5 \mathrm{~mm}$ or more), and coronoid osteophyte in all 58 elbows, loose body in $44(76 \%)$, ulnohumeral joint narrowing in 13 $(22 \%)$, and radiohumeral joint narrowing in $46(79 \%)$. Osteophytes from the medial ulnohumeral joint in anteroposterior radiograph was present in 34 elbows.

The surgical technique of UHA is almost similar to the original description [1], and we reported detailed surgical technique in the previous study [2]. Operations were performed under general anesthesia with an air tourniquet applied.

A mid-posterior longitudinal skin incision was made, the triceps muscle and posterior joint capsule were split, loose bodies and the osteophytes of the olecranon was excised, and a hole 15 to 17 in diameter opened through the olecranon fossa and the coronoid fossa.

The osteophytes of the coronoid process were excised through the opened hole, the arc of flexion-extension of the elbow was observed to be at least $10^{\circ}$ to $125^{\circ}$ after the operation. Thirty-eight cases had ulnar nerve symptoms and also had ulnar nerve neurolysis surgery.

The triceps and the skin were sutured, a plaster splint held the elbow for one day. Active and assisted exercise are begun, and discontinued when the patient is discharged from the hospital in 10 days. Outpatient's rehabilitation was continued for more 4 weeks. We analyzed the data with the Student's t-test and Chi-square test. P-values less than 0.05 were considered statistically significant.

\section{Results}

At the time of final follow-up, 41 elbows had no pain, 17 had decreased pain. The average postoperative MEPS pain scale (40 points) improved by 12 points compared to the preoperative scale. The average preoperative arc of flexion-extension of $19^{\circ}$ to $112^{\circ}$ improved to $16^{\circ}$ to $123^{\circ}$ postoperatively $(\mathrm{p}<0.001)$. The average preoperative pronationsupination arc was $84^{\circ}$ to $85^{\circ}$ which did not change $\left(85^{\circ}\right.$ to $\left.87^{\circ}\right)$.

The average post-operative MEPS function scale (22 points) improved by 6 points. Total MEPS score improved from 69 to 91 $(\mathrm{p}<0.001)$. The MEPS objective result was excellent for 40 elbows (69), good for 14 (24), fair for four (7) and no poor case.

Of 39 manual workers and 7 office workers, 35 (76) returned to their previous job or an equivalent job after the operation. About patients' satisfaction, fifty (86) were satisfactory subjective result, and eight (14) were unsatisfactory result because of pain or limited motion of the elbow. No infections, no fracture of the distal humerus, no ulnar nerve complications, and no vascular complications occurred.

Age, duration of symptom, and follow up period were not related to patients' satisfaction. Mean preoperative flexion-extension arc for satisfactory result elbows was $95^{\circ}$, while that for unsatisfactory result was $83^{\circ}$, which showed a significant difference $(\mathrm{p}<0.05)$ (Table 1$)$.

\begin{tabular}{|c|c|c|c|c|}
\hline \multicolumn{2}{|l|}{ Factor } & $\begin{array}{l}\text { Satisfactory } \\
(N=50)\end{array}$ & $\begin{array}{l}\text { Unsatisfactory } \\
(\mathrm{N}=8)\end{array}$ & p Value \\
\hline \multicolumn{2}{|c|}{ Age (yrs) } & 59 & 55 & N.S. \\
\hline \multicolumn{2}{|c|}{ Duration of symptom (mos) } & 48 & 55 & N.S. \\
\hline \multicolumn{2}{|c|}{ Follow-up period (mos) } & 42 & 49 & N.S. \\
\hline \multirow[t]{2}{*}{ MEPS } & Preop. (SD) & $70(6)$ & $67(8)$ & N.S. \\
\hline & Postop. (SD) & $94(5)$ & $75(10)$ & $<0.001$ \\
\hline
\end{tabular}

Flexion-extension arc

\begin{tabular}{|l|l|l|l|l|}
\hline & Preop. (SD) & $95^{\circ}(12)$ & $83^{\circ}(18)$ & $<0.05^{*}$ \\
\cline { 2 - 5 } & Postop. (SD) & $109^{\circ}(9)$ & $94^{\circ}(13)$ & $<0.001$ \\
\hline
\end{tabular}

$\mathrm{N}=$ number, yrs = years, $\mathrm{mos}=$ months, N.S. = no significance, $\mathrm{SD}=$ standard deviation, preop. $=$ before surgery, postop. $=$ at final follow up.

Table 1: Patients' satisfaction related to some factors.

Radiographs revealed recurrence of the coronoid osteophytes in 21 elbows, and the olecranon osteophytes in 31 elbows. Reduction of a diameter in the hole was seen in all elbows. Mean diameter in the hole in immediate postoperative anteroposterior radiograph was $15 \mathrm{~mm}$, and that at final follow up was $10 \mathrm{~mm}$.

Osteophytes from the medial ulnohumeral joint in anteroposterior radiograph, which could not be excised in this operation, was present in 34 elbows, that involved 6 unsatisfactory results (18) and MEPS pain scale of 39 points, while 24 elbows with absent medial osteophytes involved two unsatisfactory result (8) and MEPS pain scale of 40 points, which was not related to postoperative patients' satisfaction and pain scale.

Postoperative arc of flexion-extension with medial ulnohumeral osteophyte elbows was $104^{\circ}$, while that of the elbow without osteophyte was $112^{\circ}$, which showed a significant difference $(\mathrm{p}<0.01)$ (Table 2). Medial ulnohumeral osteophyte was related to decreased arc of flexion-extension but not patients' satisfaction and pain scale.

\begin{tabular}{|c|c|c|c|c|c|}
\hline \multicolumn{2}{|c|}{$\begin{array}{l}\text { Medial } \\
\text { ulnohumeral } \\
\text { osteophytes }\end{array}$} & \multirow{2}{*}{$\begin{array}{l}\text { Satisfactory } \\
(\mathrm{N}=50) \\
28\end{array}$} & \multirow{2}{*}{$\begin{array}{l}\text { Unsatisfactory } \\
(\mathbf{N}=\mathbf{8}) \\
6(18)\end{array}$} & \multirow{2}{*}{$\begin{array}{l}\text { MEPS } \\
\text { pain } \\
\text { scale }\end{array}$} & \multirow{2}{*}{$\begin{array}{l}\text { Flexext. } \\
\text { arc (SD) } \\
104^{\circ}(11)\end{array}$} \\
\hline Present & $(\mathrm{N}=34)$ & & & & \\
\hline Absent & $(\mathrm{N}=24)$ & 22 & $2(8)$ & 40 & $112^{\circ}(7)$ \\
\hline \multicolumn{3}{|c|}{ Significant difference } & N.S. & N.S. & $P<0.01$ \\
\hline
\end{tabular}

Table 2: Medial ulnohumeral osteophyte related to satisfaction and motion.

\section{Illustrative Case}

A 59-year-old mechanic man had motion pain and limited motion of his right elbow. Flexion-extension arc of his right elbow was $30^{\circ}$ to $105^{\circ}$, total MEPS score was 70 . Preoperative radiograph showed osteophytes in olecranon, medial or tip of coronoid, and radial head (Figure 1). Thirty months after UHA, he had no elbow pain, flexionextension arc was $15^{\circ}$ to $120^{\circ}$, and MEPS was 95 with excellent and 
Citation: Nobuta S, Sato K, Itoi E (2018) Influence of Remained Medial Osteophyte on the Outcome after Ulnohumeral Arthroplasty. J Arthritis 7:

Page 3 of 4

satisfactory result. Postoperative radiograph revealed osteophyte resection with observation of the hole, and remained medial ulnohumeral osteophyte (Figure 2).
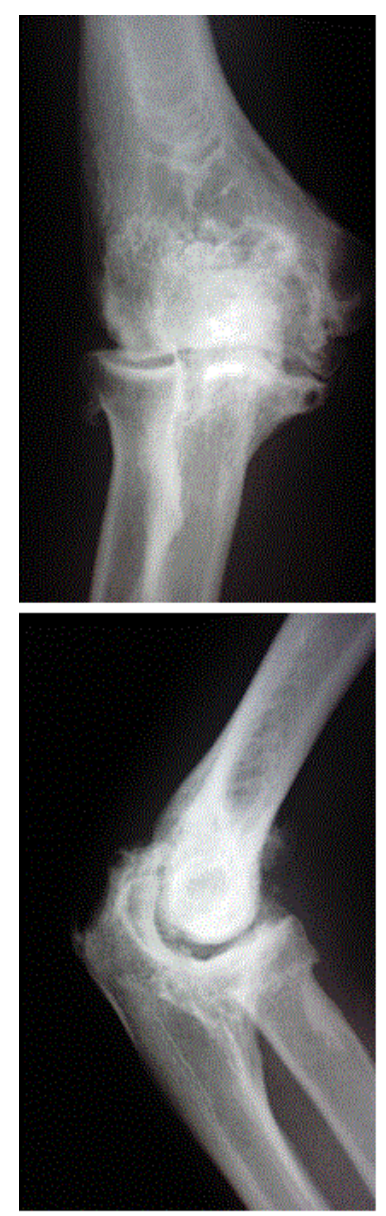

Figure 1: A 59-year-old man, preoperative (A) AP and (B) lateral radiographs of right elbow show osteophytes in olecranon, medial and the tip of coronoid, and radial head.

\section{Discussion}

Ulnohumeral arthroplasty (UHA) was established by Kashiwagi [1], and the concept of the operation is to remove the part of the ulnohumeral joint causing functional disability. Its main advantage is to access to both the posterior and anterior parts of the elbow joint, simple and safe technique, and simple postoperative rehabilitation $[7,8]$. In our series of 58 elbows, 41 (71) had complete relief of pain, 17 (29) had incomplete relief, and the flexion-extension arc improved by $14^{\circ}$.

Mean MEPS score improved from 69 to 91, and MEPS evaluation was excellent in 40, good in 14, and fair in four. Subjectively, 50 were satisfactory result and 8 were unsatisfactory. These results are almost similar to those reported in the literature [6-12]. In our series, eight elbows were unsatisfactory results because of pain or limited motion of the elbow. The mean preoperative flexion-extension arc was significantly greater in elbows with satisfactory results than those with unsatisfactory results. Therefore, we believe that flexion-extension arc is a predictive factor of postoperative outcome.
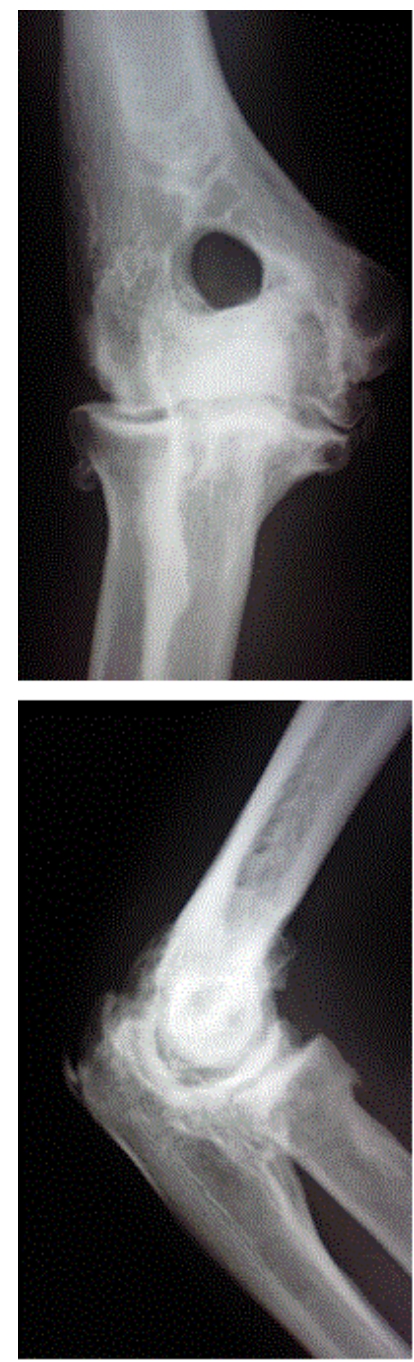

Figure 2: At final follow up (thirty months after operation), postoperative (A) AP and lateral radiographs reveal adequate osteophyte resection with the hole, and remained medial ulnohumeral osteophyte, with excellent and satisfactory result.

The surgical technique of UHA does not allow access to the medial ulnohumeral osteophyte and to the radiohumeral joint. This is considered to be disadvantages of this surgical procedure. However, the influence of remained medial ulnohumeral osteophyte on the outcome is still unclear. Minami [3] and Oka et al. [4] reported that the main lesions of elbow osteoarthritis are present at the medial ulnohumeral joint which cause pain and limitation of motion. In our series, remained medial ulnohumeral osteophyte influenced the flexionextension arc but not patients' satisfaction and pain scale (Table 2). Open debridement using a medial or lateral approach has been reported [13-15], and these allow management of the medial ulnohumeral osteophyte. However, these require extensive soft tissue dissection including a danger of dividing collateral ligament. We consider that medial approach is not so valuable, because remained medial ulnohumeral osteophyte did not influence patients' satisfaction 
Citation: Nobuta S, Sato K, Itoi E (2018) Influence of Remained Medial Osteophyte on the Outcome after Ulnohumeral Arthroplasty. J Arthritis 7:

Page 4 of 4

and pain scale. Furthermore, we consider that UHA is indicated with terminal motion pain, osteophytes located in the olecranon or coronoid process or olecranon fossa and medial ulnohumeral joint, preoperative flexion-extension arc was $90^{\circ}$ or more, and that it is contraindicated when patients have continuous motion pain of the elbow with severe involvement of the radiohumeral joint.

\section{Conclusion}

The ulnohumeral arthroplasty is useful to reduce pain and to increase the range of motion for elbow osteoarthritis, and it is contraindicated with severe involvement of the radiohumeral joint. Remained medial ulnohumeral osteophyte is related to decreased arc of flexion-extension but not patients' satisfaction and pain scale.

\section{Conflict of Interests}

The authors report no conflict of interests.

\section{Acknowledgment}

The authors wish to thank Ms. Yumi Watabe and Ms. Hiromi Takeda, Tohoku Rosai Hospital, for assisting in the manuscript preparation.

\section{References}

1. Kashiwagi D (1998) Osteoarthritis and Outerbridge-Kashiwagi method (in Japanese). In Orthopaedic Mook 54. Kanehara, Tokyo, Japan,

2. Nobuta S, Sato K, Itoi E (2016) Long-term Results of Ulnohumeral Arthroplasty for Symptomatic Elbow Osteoarthritis. J Arthritis 5: 196.

3. Minami M (1997) Roentgenological studies of osteoarthritis of the elbow joint. J Jpn Orthop Assoc 5: 1223-1236.
4. Oka Y, Ohta K, Saitoh I (1998) Debridement arthroplasty for osteoarthritis of the elbow. Clin Orthop 351: 127-134.

5. Morrey BF (2009) Functional evaluation of the elbow. In The Elbow and Its Disorders, 4th ed. Saunders Elsevier, Philadelphia, PA, USA.

6. Vingerhoeds B, Degreef I, De Smet L (2004) Debridement arthroplasty for osteoarthritis of the elbow (Outerbridge-Kashiwagi procedure). Acta Orthop Belg 70: 306-310.

7. Morrey BF (1992) Primary degenerative arthritis of the elbow. Treatment by ulnohumeral arthroplasty. J Bone Joint Surg Br 74: 409-413.

8. Minami M, Kato S, Kashiwagi D (1996) Outerbridge-Kashiwagi's method for arthroplasty of osteoarthritis of the elbow - 44 elbow followed for 8 16 years. J Orthop Sci 1: 11-15.

9. Antuna SA, Morrey BF, Adams RA, O’Driscoll SW (2002) Ulnohumeral arthroplasty for primary degenerative arthritis of the elbow: Long-term outcome and complications. J Bone Joint Surg Am 84: 2168-2173.

10. Phillips NJ, Ali A, Stanley D (2003) Treatment of primary degenerative arthritis of the elbow by ulnohumeral arthroplasty. J Bone Joint Surg $\mathrm{Br}$ 85: 347-350.

11. Sarris I, Riano FA, Goebel F, Goitz RJ, Sotereanos DG (2004) Ulnohumeral arthroplasty: results in primary degenerative arthritis of the elbow. Clin Orthop Relat Res 420: 190-193.

12. Tashjian RZ, Wolf JM, Ritter M, Weiss AP, Green A (2006) Functional outcomes and general health status after ulnohumeral arthroplasty for primary degenerative arthritis of the elbow. J Shoulder Elbow Surg 15: 357-366.

13. Tsuge K, Murakami Y, Yasunaga Y, Kanaujia RR (1987) Arthroplasty of the elbow. J Bone Joint Surg Br 69: 116-120.

14. Oka Y (2000) Debridement arthroplasty for osteoarthritis of the elbow. 50 patients followed mean 5 years. Acta Orthop Scand 71: 185-190.

15. Wada T, Isogai S, Ishii S, Yamashita T (2005) Debridement arthroplasty for primary osteoarthritis of the elbow. Surgical technique. J Bone Joint Surg Am 87: 95-105. 